\title{
La desigualdad social en Chihuahua: implicaciones en la educación básica en un horizonte de doce años
}

\author{
Ricardo Almeida Uranga \\ Universidad Autónoma de Ciudad Juárez \\ Instituto de Ciencias Sociales y Administración
}

\section{Resumen}

$\tau$

oera (1996) realizó un primer diagnóstico del sistema de educación estatal con datos de primaria y secundaria del ciclo escolar 1994-95 sobre el grado de asociación ( $r$ de Pearson y coeficiente de determinación) entre la eficiencia terminal, la reprobación y deserción en educación básica (primaria y secundaria), y la absorción (de secundaria) versus el índice de marginalidad en los 67 municipios del estado de Chihuahua. Se realizó un segundo análisis de correlación con datos correspondientes al ciclo escolar 2006-2007. En ambos ciclos escolares hay una alta asociación entre marginalidad municipal y los indicadores educativos que se emplearon, lo que corrobora la tesis reproduccionista

en educación (Bourdieu y Passeron, 1973, 1977; Bowles y Gintis, 1985). Las asociaciones se dan en este sentido: a mayor marginalidad municipal, menor eficiencia terminal, mayor tasa de reprobación y mayor deserción en primaria. Los indicadores de secundaria muestran asociaciones inexistentes y no resultan ser significativas. Con base en los resultados se recomiendan orientaciones en la política y en el diseño de programas y proyectos públicos de educación para contrarrestar el determinismo contextual en los resultados educativos.

Palabras clave: administración pública, análisis estadístico, cambio educacional, deserción, discriminación social. 
REVISTA DE INVESTIGACIÓN EDUCATIVA DE LA REDIECH N. 7 ISSN: 2007-4336

\section{Contextualización y método}

\section{Ubicación geográfica del estado de Chihuahua}

De acuerdo a la información que proporciona la Secretaría del Medio Ambiente y Recursos Naturales (SEmarnat, diciembre 2007) el estado de Chihuahua se localiza al norte de la República Mexicana. Chihuahua es el estado con mayor extensión territorial en el país, equivalente a 247,087 $\mathrm{km}^{2}$, que representa el $12.56 \%$ del territorio nacional.

\section{Encuadre general del estudio}

Un estudio en el área educativa que analiza las asociaciones entre indicadores educativos e indicadores socioeconómicos (como lo es el índice de marginalidad municipal) se circunscribe dentro de la perspectiva reproduccionista en educación (Baudelot y Establet, 1971; Bourdieu y Passeron, 1973,1977; Bowles y Gintis, 1985), este estudio se aborda desde los campos disciplinares tanto de la sociología como de la educación. Las reformas sociales y educativas que reflejaron el espíritu de la década de los sesenta buscaron la transformación social a través de la acción educativa. La apuesta fue ofrecer una educación de calidad con igualdad de oportunidad de acceso, permanencia y logro para los diversos grupos y estratos sociales esperando lograr con ello un impacto para disminuir las brechas socioeconómicas de la población atendida (Aguilar, 2002; Connell, 1997). La perspectiva reproduccionista de los estudios en educación ha encontrado evidencia de que esta apuesta sociopolítica no ha tenido de manera predominante los resultados que se buscaron. Asimismo, dicha perspectiva ha elaborado diversas interpretaciones teóricas alrededor de la función reproductiva de la educación (Elboj, García y Guarro, 2005) tal como la de Bourdieu (1977) que estudió la dinámica de la transmisión cultural a través del sistema educativo y de cómo determinados grupos sociales tienen garantizado el éxito o el fracaso escolar de manera discriminativa. Otra elaboración teórica relacionada con esta perspectiva es la que realizaron Baudelot y Establet (1971) en la que ofrecen explicaciones acerca de como la división del trabajo en la sociedad corresponde a una división social producida por el propio sistema educativo.

\section{Los estudios empíricos y de corte cuantitativo}

Dentro de esta vertiente se llevan a cabo estudios realizando análisis de asociación lineal entre indicadores de desempeño del sistema educativo (Secretaría de Educación y Cultura, 2010) e indicadores socioeconómicos (Correa y Restrepo, 2002; Hernández, Fernández-Collado y Baptista, 2010). El presente análisis se inscribe dentro de dicha perspectiva.

\section{Antecedentes}

El Gobierno del Estado da origen a la Coordinación de Investigación y Desarrollo Académico en 1996 (CIDA). Este organismo surge como consecuencia de una coyuntura donde convergen los siguientes procesos: a) la crisis del sistema político mexicano; b) un proceso de alternancia política; c) las consecuencias del conflicto entre el gobierno estatal de extracción pa- 
nista y la dirigencia magisterial; y d) el inicio del nuevo pacto para transferir la parte federal del sistema educativo al gobierno estatal. A raíz de lo anterior, Loera (1996) realizó un primer diagnóstico del sistema de educación estatal con datos de primaria y secundaria del ciclo escolar 1994-95 sobre el grado de asociación ( $r$ de Pearson y coeficiente de determinación) entre la eficiencia terminal, la reprobación y deserción de educación básica (primaria y secundaria) y la absorción (de secundaria), versus el índice de marginalidad en los 67 municipios del estado de Chihuahua.

Los indicadores educativos que se utilizaron en el análisis publicado en 1996 por Loera correspondientes al nivel de primaria fueron: la eficiencia terminal, la deserción y la reprobación. Se obtuvieron los grados de asociación y los coeficientes de determinación de los indicadores educativos, desagregados a nivel municipal, con el índice de marginalidad municipal (IMM) desarrollado por el Consejo Nacional de Población (Conapo, 1990). Para su análisis, Loera (1996) empleó datos de inicio de cursos dados a conocer por la Secretaría de Educación Pública (SEP) en el ciclo escolar 1994-1995.

\section{Fuentes de información y análisis de los datos}

En el presente análisis se lleva a cabo un contraste de los principales hallazgos y conclusiones alcanzados en el estudio mencionado (Loera, 1996). Para ello se utilizan los mismos indicadores correspondientes a la información proporcionada por la SEP también de inicio de cursos, pero en esta ocasión del ciclo escolar 2006-2007.
Es decir, doce años después. Nuevamente se calculan los grados de asociación y los coeficientes de determinación entre los indicadores educativos y el IMM con datos del Conapo (2000). Esto facilita realizar el contraste entre los resultados obtenidos para el ciclo escolar 2006-2007 con el informe presentado por Loera en 1996, con datos del ciclo escolar 1994-1995. Esta contrastación constituye un primer momento de un seguimiento longitudinal que abarca otros dos ciclos escolares: 2009-2010 y 2014-2015 (la información de este ciclo se generará hasta la segunda mitad del año 2015). Por razones de extensión, en este trabajo se presentan únicamente los resultados del primer ciclo escolar considerado (2006-2007).

\section{Asociación entre marginalidad municipal y educación}

\section{Resultados}

En el presente análisis se contrastan los resultados de las diferentes asociaciones obtenidas previamente para el ciclo escolar 1994-1995 (Loera, 1996) con la información correspondiente al ciclo escolar 20062007 (ver Tablas 1 a la 3). Para la apreciación de los diferentes niveles de asociación se consideran fuertes las mayores a .60; débiles a las asociaciones entre .59 y .24 ; e inexistentes, a las menores de .24. Para las asociaciones del ciclo escolar 2006-2007, dos asteriscos $\left.{ }^{* *}\right)$ indican un nivel de significancia estadística de .01; un asterisco $\left.{ }^{*}\right)$ indica un nivel de significancia estadística de .05 .

La marginalidad municipal se relaciona de la siguiente manera con los indicadores que se mencionan (ver tabla 1): 
A mayor marginalidad menor eficiencia terminal de primaria en ambos ciclos escolares considerados. La asociación es fuerte y significativa. La variabilidad de la eficiencia terminal es explicada en un $61 \%$ para los datos de 1994-1995 y en un 46.4\% para los datos del ciclo escolar 2006-2007.

A mayor marginalidad mayor tasa de reprobación de la primaria. La asociación es fuerte y significativa para ambos ciclos escolares considerados. De hecho, el nivel de asociación y el coeficiente de determinación son iguales para ambos ciclos escolares: .76 y .58 respectivamente.

A mayor marginalidad municipal mayor deserción de la primaria. El nivel de asociación es débil y significativo. La variabilidad de la tasa de deserción en el ciclo escolar 1994-1995 se explica por la marginalidad municipal en un 33\% y en el ciclo escolar 2006-2007 en un 16.8\%. Es decir, se explica en menor proporción la variabilidad de la deserción en primaria en el ciclo escolar 2006-2007.

La tasa de absorción en secundaria se encuentra negativamente asociada con la marginalidad municipal. La asociación es débil y significativa. El nivel de asociación lineal entre ambas variables se ha mantenido en el mismo nivel entre 1994 y 2007.

La marginalidad municipal se encuentra relacionada a nivel de primaria con menor tasa de alumnos por grupo y menor tasa de alumnos por escuela. Estos niveles de asociación son fuertes y significativos en ambos ciclos escolares considerados. La asociación fue ligeramente más alta en el ciclo escolar 2006-2007. La variabilidad de la tasa de alumnos por grupo en primaria se explica en un $45 \%$ por la marginalidad municipal en el ciclo escolar 1994-1995 y en un 50\% en el ciclo escolar 2006-2007. La variabilidad de la tasa de alumnos por escuela se explica en un $44 \%$ por la marginalidad municipal en el ciclo escolar 19941995 y se explica en la misma proporción doce años después. Lo anterior es indicativo de que en las zonas más pobres del estado hay menor población y mayor dispersión de la misma.

La asociación entre la tasa de alumnos por maestro en primaria y la marginalidad municipal es débil y significativa en ambos ciclos escolares considerados. El porcentaje de variabilidad explicado por la marginalidad es muy baja, el 15\% en el ciclo escolar 1994-1994 y el 23\% en el ciclo escolar 2006-2007.

Los indicadores de secundaria muestran asociaciones inexistentes y no resultan ser significativas con la marginalidad de los municipios. Esto puede ser indicativo de una mayor capacidad del sistema escolar para ofrecer iguales oportunidades de logro, permanencia y egreso de los estudiantes de este nivel. Es decir, hay una disociación entre las condiciones de marginalidad municipal por un lado y los indicadores de comportamiento del sistema educativo a nivel de secundaria por otro. 


\begin{tabular}{|c|c|c|c|c|}
\hline \multicolumn{5}{|c|}{$\begin{array}{c}\text { Tabla } 1 \\
\text { Indicadores educativos asociados con el índice de marginalidad municipal }\end{array}$} \\
\hline & \multicolumn{2}{|c|}{ Coeficiente de correlación } & \multicolumn{2}{|c|}{$\begin{array}{l}\text { Coeficiente de } \\
\text { determinación }\end{array}$} \\
\hline & $\begin{array}{c}\text { Ciclo } \\
\text { escolar } \\
1994-95\end{array}$ & $\begin{array}{l}\text { Ciclo escolar } \\
2006-07\end{array}$ & $\begin{array}{c}\text { Ciclo } \\
\text { escolar } \\
1994-95\end{array}$ & $\begin{array}{c}\text { Ciclo } \\
\text { escolar } \\
2006-07\end{array}$ \\
\hline 1. Tasa de eficiencia terminal de la primaria & -0.78 & $-.688(* *)$ & 0.61 & .464 \\
\hline 2. Tasa de reprobación de la primaria & 0.76 & $.769\left(^{* *}\right)$ & 0.58 & .586 \\
\hline 3. Tasa de alumnos por grupo en primaria & -0.67 & $-.712(* *)$ & 0.45 & .500 \\
\hline 4. Tasa de alumnos por escuela en primaria & -0.66 & $-.670(* *)$ & 0.44 & .441 \\
\hline 5. Tasa de deserción de la primaria & 0.58 & $.429(* *)$ & 0.33 & .168 \\
\hline 6. Tasa de absorción de la secundaria & -0.53 & $-.595(* *)$ & 0.28 & .341 \\
\hline 7. Tasa de alumnos por maestro en primaria & -0.38 & $-.494(* *)$ & 0.15 & .232 \\
\hline 8. \% de la población estatal que vive en el municipio & -0.24 & -.231 & 0.05 & .039 \\
\hline 9. Tasa de reprobación de la secundaria & -0.09 & -.224 & 0.12 & .036 \\
\hline 10. Tasa de deserción en secundaria & -0.09 & .226 & 0.009 & .036 \\
\hline 11. Tasa de eficiencia terminal de la secundaria & 0.02 & -.122 & 0.0004 & -.001 \\
\hline
\end{tabular}

En primaria, la reprobación y la deserción se encuentran inversamente asociadas con el nivel de eficiencia terminal (ver tabla 2). Es decir, a mayor reprobación y a mayor deserción, menor eficiencia terminal de primaria. Esta asociación es fuerte y significativa en ambos ciclos escolares. Sin embargo, la asociación de reprobación (-0.74 en 1994-1995 y -0.62 en 2006-2007) es mas alta que la de deserción (-0.62 y -0.60 respectivamente). Esto indica que una menor eficiencia terminal está más fuertemente determinada por la reprobación que por la deserción.

También se puede apreciar en la tabla 2 que a mayor número de alumnos por escuela y por grupo, la eficiencia terminal es mayor. Esta asociación en el ciclo escolar 1994-1995 fue fuerte y en el ciclo escolar
2006-2007 se considera débil y significativa.

Dicha asociación perdió fuerza en el transcurso de 12 años, al igual que el porcentaje de la población estatal que vive en el municipio (0.29 y .183 respectivamente) y su asociación con la tasa de eficiencia terminal de primaria. Esto indica que el tamaño de la población estudiantil en las escuelas, el tamaño de los grupos y la cantidad de población viviendo en el municipio son variables que han perdido asociación lineal con la eficiencia terminal. Esto ocurre seguramente porque se han establecido dinámicas distintas al interior de las escuelas y de las aulas, que hacen independientes el número de estudiantes de su permanencia y terminación del nivel de estudios de primaria. 
REVISTA DE INVESTIGACIÓN EDUCATIVA DE LA REDIECH N. 7

ISSN: 2007-4336

\begin{tabular}{|c|c|c|}
\hline \multicolumn{3}{|c|}{ Tabla 2} \\
\hline Indicadores educativos asociados con I & \multicolumn{2}{|c|}{ Correlación } \\
\hline & Ciclo escolar & Ciclo escolar \\
\hline & 1994-1995 & $2006-2007$ \\
\hline 1. Índice de marginalidad municipal & -0.78 & $-.688(* *)$ \\
\hline 2. Tasa de reprobación de primaria & -0.74 & $-.628(* *)$ \\
\hline 3. Tasa de alumnos por escuela & 0.68 & $.463(* *)$ \\
\hline 4. Tasa de alumnos por grupo & 0.65 & $.428\left(^{* *}\right)$ \\
\hline 5. Tasa de deserción de la primaria & -0.62 & $-.605(* *)$ \\
\hline 6. Tasa de absorción de la secundaria & 0.45 & $.388(* *)$ \\
\hline 7. Tasa de alumnos por maestro & -0.30 & $.323\left(^{*}\right)$ \\
\hline 8. \% de la población estatal que viven en el municipio & 0.29 & .183 \\
\hline 9. Tasa de reprobación de secundaria & 0.28 & .151 \\
\hline 10. Número de escuelas de primaria & 0.21 & .052 \\
\hline 11. Tasa de eficiencia terminal de secundaria & 0.06 & .093 \\
\hline 12. Tasa de deserción de secundaria & -0.04 & $-.373(* *)$ \\
\hline
\end{tabular}

Tabla 3

Indicadores educativos asociados con la tasa de eficiencia terminal de secundaria

Correlación

\begin{tabular}{lcc} 
& \multicolumn{2}{c}{ Correlación } \\
\cline { 2 - 3 } & $\begin{array}{c}\text { Ciclo escolar } \\
1994-1995\end{array}$ & $\begin{array}{c}\text { Ciclo escolar } \\
2006-2007\end{array}$ \\
\hline 1. Tasa de alumno por maestro & 0.31 & -0.155 \\
2. Tasa de deserción de secundaria & -0.30 & $-.606(* *)$ \\
3. Tasa de alumnos por grupo & 0.13 & -.048 \\
4. Tasa de alumnos por escuela & 0.13 & .023 \\
5. \% de la población estatal que vive en el municipio & 0.09 & .059 \\
6. Tasa de absorción de la secundaria & 0.08 & .187 \\
7. Tasa de eficiencia terminal de la primaria & 0.06 & .093 \\
8. Tasa de deserción de la primaria & -0.04 & .092 \\
9. Número de escuelas primarias & 0.03 & .012 \\
10. Índice de marginalidad municipal & 0.02 & -.122 \\
11. Tasa de reprobación de secundaria & -0.02 & -.312 \\
12. Tasa de reprobación de primaria & -0.01 & -.052 \\
\hline
\end{tabular}


Los índices de asociación que se muestran en la tabla 3 tienen que ver con una serie de indicadores educativos y de población. Ninguno, a excepción del que se comenta a continuación muestran algún tipo de asociación con la eficiencia terminal de secundaria. En el ciclo escolar 2006-2007, muestra una asociación fuerte la deserción de secundaria con la eficiencia terminal del mismo nivel. La relación es inversa. A mayor deserción durante el ciclo escolar, la eficiencia terminal es menor. El grado de asociación lineal entre ambas variables se incrementó al doble en el lapso de los doce años entre los dos ciclos escolares considerados. La tasa de reprobación, en cambio, del ciclo escolar 2006-2007 se considera débil y equivale a la mitad que la asociación de deserción y eficiencia terminal. Nuevamente, a nivel de secundaria la dinámica entre indicadores es distinta que en primaria. En este nivel, la deserción es una variable más fuertemente asociada con la eficiencia terminal que la reprobación.

\section{Conclusión}

En los dos periodos considerados para el análisis de los datos (1994-95 y 2006-07) se hace evidente que el contexto socio-económico tiene una influencia determinante en los resultados educativos en el estado de Chihuahua y que esta condición se mantiene de manera inercial. Es necesaria una revisión profunda de la política educativa, de los programas y proyectos educativos para que el sistema educativo estatal mejore el nivel de impacto de sus procesos de enseñanza de manera mayormente igualitaria en los diferentes segmentos de la población en el estado.

\section{Agradecimientos}

Se agradece el apoyo recibido por las siguientes instancias:

Secretaría de Educación Pública (Fondo Sectorial 2007).

Subsecretaría de Educación Básica (Fondo Sectorial 2007).

Consejo Nacional de Ciencia y Tecnología (SNI 2008-2010 y 2014-2016)

Universidad Autónoma de Ciudad Juárez (Programa de Estímulos 2007- 2014).

Dichos respaldos se han utilizado para realizar el proyecto de investigación titulado: "El estado de la educación en Chihuahua en 1997 y en el 2013: avances, retrocesos y propuestas". El presente reporte es un producto parcial de dicho proyecto.

\section{Referencias}

Aguilar, M. (2002). La educación en México (1970-2000): de una estrategia Nacional a una estrategia Regional. Recuperada el 7 de abril de 2010 en: http://www.latarea.com.mx/index.htm/

Baudelot, Ch. y R. Establet. (1976). La Escuela Capitalista en Francia. Siglo XXI. Madrid, España.

Bourdieu, P. y Passeron, J.C. (1973). Los Estudiantes y la Cultura. Labor. Buenos Aires, Argentina.

Bourdieu, P. y. Passeron, J.C. (1977). Los Estudiantes y la Cultura. Laia. Barcelona, España.

Bowles, S. y Gintis, H. (1973). La Instrucción Escolar en la América Capitalista. Labor. Buenos Aires, Argentina.

Connell, R. W. (1977). Escuelas y Justicia Social. Morata. Madrid.

Correa, S., Puerta, A. y Restrepo, B. (2002). Investigación Evaluativa: Especialización en Teoría, Métodos y Técnicas de Investigación Social. Instituto Colombiano para el Fomento de la Educación Superior. Bogotá.

Elboj Saso, C., García Yeste, C. y Guarro Pallás, A. (2005). "La Sociología ante las Prácticas Escolares Transformadoras". Presentación en la XI Conferencia de Sociología de la Educación. Santander, España.

Esquivel, G. (2009). "El Mercado Laboral Rural en México: Caracterización y Agenda de Investigación”. Naciones Unidas Comisión Económica para América 
Latina y el Caribe - CEPAL. (Distribución limitada) 1-101.

Hernández Sampieri, R., Fernández-Collado, C. y Baptista Lucio, P. (2010). Metodología de la Investigación (5a ed.). Mc-Graw-Hill Interamericana. México.

Loera, A. (1996). Educación Básica y Marginalidad Municipal. Foro 2, 3 , 3-6.

Loera, A. (1998). Fijan Chihuahuenses sus Bases Educativas. Foro 21, 11, 1.

Martínez Pérez, L. (1994). Reseña de Chihuahua: Historia de una Alternativa de Alberto Aziz Nassif. Perfiles Latinoamericanos, 005, 167-169.

Ordaz Díaz, J. L. (2009). México: Impacto de la Educación en la Pobreza Rural. Sede Subregional de la CEPAL en México - Serie Estudios y Perspectivas, 105, 1-40.

Ornelas, C. (1995). El Sistema Educativo Mexicano. Centro de Investigación y Docencia Económicas,
Nacional Financiera, Fondo de Cultura Económica. México.

Pardo, M. del C. (coord.). (1999). Federalización e Innovación Educativa en México. El Colegio de México. México.

Secretaría de Educación Pública. (2006). Sistema de Indicadores Educativos de los Estados Unidos Mexicanos. Conjunto Básico para el Ciclo Escolar 2004-2005. $1^{a}$ ed. Dirección General de Planeación y Programación, SEP, Instituto Nacional para la Evaluación de la Educación. México.

Secretaría de Educación y Cultura. (2010). Servicios Educativos del Estado de Chihuahua, Indicadores educativos 2005-2006. Recuperado el 15 de enero de 2011 en: http://seech.gob.mx/

SEMARNAT. (2007) Mi Chihuahua Hoy. Monografía del Estado. Chihuahua, Chih. México 1995-96. Recuperado el 13 de diciembre de 2007 en: www.semarnat.gob.mx. 